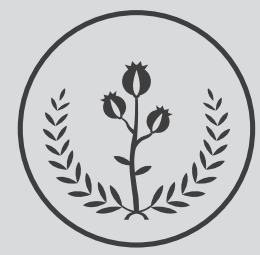

FUCS

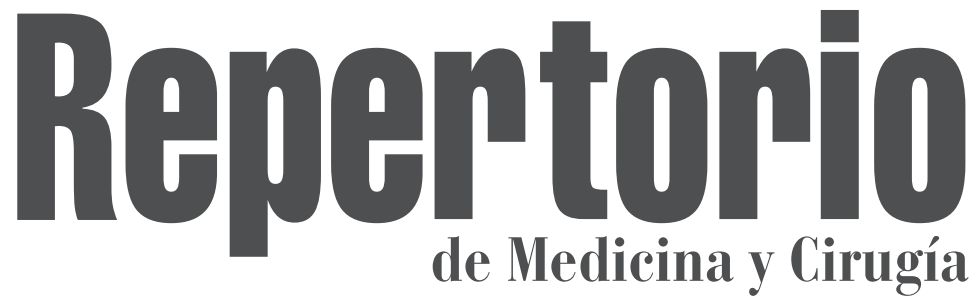

ISSN: 0121-7372 • ISSN electrónico: 2462-991X

Artículo de revisión

\title{
Manejo de la sepsis en el embarazo. Revisión de la literatura
}

Favian Leonardo Medina MD $^{\mathrm{a}}$ Iván Camilo González MD $^{\mathrm{b}}$

\section{Management of sepsis in pregnancy: a review of the literature}

${ }^{a}$ Medicina de Urgencias, Medicina Crítica y Cuidado Intensivo. Fundación Universitaria de Ciencias de la Salud. Bogotá DC, Colombia.

${ }^{b}$ Medicina de Urgencias, Fundación Universitaria de Ciencias de la Salud. Bogotá DC, Colombia, Fundación Universitaria San Martin, Epidemiología, Universidad de Boyacá, Colombia.

\section{R E S U M EN}

Introducción: la sepsis se reconoce como una enfermedad común y mortal, cuya epidemiología obliga al médico tratante a realizar un diagnóstico oportuno por el alto riesgo de complicaciones o muerte. En las gestantes hay una gran vulnerabilidad debido a los cambios físicos y hormonales que están afrontado. Es indispensable iniciar el tratamiento tan pronto se tenga el diagnóstico, para evitar que sea mortal tanto para la madre como para el feto. Objetivo: es por esto que el objetivo es una revisión sobre la intervención en las pacientes con diagnóstico de sepsis en estado de embarazo al ingreso a la UCI. Discusión: se consideraron los siguientes ejes temáticos: definición clara de sepsis en el embarazo, factores de riesgo, intervención terapéutica en UCI y consecuencias para el feto durante su intervención. Conclusiones: se concluye que el manejo de la sepsis en las embarazadas es muy similar a las que no lo están. Hacen falta investigaciones con evidencia científica, velando siempre por la vida ya que no solo se trata un ser humano sino de dos.

Palabras clave: sepsis obstétrica; infección; cuidado crítico; tratamiento oportuno.

(C) 2021 Fundación Universitaria de Ciencias de la Salud - FUCS. Este es un artículo Open Access bajo la licencia CC BY-NC-ND (http://creativecommons.org/licenses/by-nc-nd/4.0/).

\section{INFORMACIÓN DEL ARTÍCULO}

Historia del artículo:

Fecha recibido: junio 27 de 2019

Fecha aceptado: septiembre 9 de 2019
Autor para correspondencia.

Dr. Iván Camilo González

Icgonzalezc@fucsalud.edu.co
DOI

10.31260/RepertMedCir.01217273.221 
Sepsis is considered a common condition and a major cause of death. Due to its epidemiologic features the treating physician must make a timely diagnosis for patients are at a high risk of developing complications or dying. Pregnant women are more vulnerable to infection due to the physiologic and hormonal changes they undergo. Early initiation of treatment when sepsis is recognized is critical to prevent mother or fetal death. Thus, the objective of this study was to conduct a review of interventions in pregnant patients with sepsis admitted to the ICU, using the following search headings: clear definition of sepsis in pregnancy, risk factors, therapeutic intervention at the ICU and consequences for the fetus during the intervention. We concluded that the management of sepsis in pregnant women is very similar to that in non-gravid women. There is a lack of evidenced-based studies focused in always protecting life for it implies the preservation of not only one human life but two lives.

Key words: sepsis in obstetrics; infection; critical care; timely treatment.

(C) 2021 Fundación Universitaria de Ciencias de la Salud - FUCS. This is an open access article under the CC BY-NC-ND license (http://creativecommons.org/licenses/by-nc-nd/4.0/).

\section{INTRODUCCIÓN}

La sepsis se define como la respuesta del huésped ante una infección por un tipo de microorganismo, lo cual conlleva a una serie de procesos fisiopatológicos caracterizados por distintos tipos de células que se ven afectadas en el proceso. Estas alteraciones se reflejan en los sistemas orgánicos y funcionales, con una alta prevalencia de mortalidad y morbilidad en las pacientes que la desarrollan. ${ }^{1}$ Es importante aclarar que su definición ha variado con el paso del tiempo debido a su identificación y tratamiento. Una de las que más tuvo auge fue la desarrollada por la Society of Critical Care Medicine en 2003, que la define como una de las causas más frecuentes de ingreso a la unidad de cuidados intensivos, siendo esta la primera causa de muerte. ${ }^{2}$ Se considera que se presenta en forma severa cuando ocurre una o más disfunciones de los diferentes sistemas. Es poca la literatura y la información disponibles y en su mayoría se basan en casos clínicos o estudios retrospectivos con muestras pequeñas. Aquí cabe resaltar que esta es una de las razones por la cual se ve la necesidad de ver la sepsis en el embarazo como un tema importante para investigación, buscando siempre un mejor tratamiento. ${ }^{3}$

Constituye un gran reto su diagnóstico y tratamiento cuando son pacientes en estado de embarazo, debido a que pueden evolucionar a un shock séptico o a una disfunción orgánica múltiple causando la muerte materna y fetal en cifras que oscilan entre 27 y $59 \% .^{4,5}$

Los factores que más inciden en su aparición se asocian con el aumento de las comorbilidades asociadas, los procedimientos invasivos, trasplantes y/o inmunosupresión. Las infecciones bacterianas encabezan la lista de estos factores, siendo una de las causas de mortalidad más importantes en las unidades de cuidados intensivos, con un costo anual de 17 mil millones de dólares aproximadamente. ${ }^{3,6}$

En México entre 2005 y 2015 se evidenció en las unidades de cuidados intensivos obstétricos que era una de las causas más comunes de muerte en maternas, en la mayoría secundaria a todas las infecciones del parto, donde los mayores causantes son Eschericha coli, Klebsiella pneumoniae, Enterobacter sp y Enterococcus faecalis. Se sabe que la causa polimicrobiana es común y los focos son de origen pélvico ${ }^{2}$ del tracto urinario, el respiratorio y el abdomen, que corresponden a endometritis, sepsis puerperal, pielonefritis y neumonía. ${ }^{7}$ Se pueden nombrar tres condiciones que pueden ser claves para que las muertes maternas no sigan en aumento: la vigilancia prenatal temprana que genere de manera idónea la identificación y el diagnóstico oportuno en las pacientes de alto riesgo que puedan desarrollar complicaciones, en segundo término vigilar que la asistencia a las maternas que se encuentran hospitalizadas sea de alta calidad y por último la información clara a las pacientes para que identifiquen los signos y síntomas de alarma. ${ }^{8,9}$

En Estados Unidos 5\% de las muertes maternas ocurren por sepsis, viéndose reflejado de la siguiente manera: de 100.000 mujeres el $28.47 \%$ entre las edades de 15 a 49 años fallecen por septicemia, evidenciándose que la incidencia va en aumento. ${ }^{10}$ Sin embargo, es importante resaltar que el tratamiento oportuno, adecuado y temprano en las unidades de cuidados intensivos obstétricos cursa con una reducción de la morbilidad y mortalidad; este acontecimiento llevó al desarrollo de "sobreviviendo a la sepsis", una de las campañas que adelanta más de un hospital de Estados Unidos con el fin de realizar una identificación temprana de la enfermedad. ${ }^{11,12}$

El tratamiento adecuado y oportuno es una prioridad para preservar la salud materna y fetal. Dado lo anterior y evidenciando que no existen estudios bien soportados sobre el manejo de la sepsis en el embarazo, existe la necesidad de realizar modificaciones a su tratamiento teniendo en cuenta como base los cambios fisiológicos del embarazo así como el inicio del tratamiento con la ingestión de líquidos y de un antimicrobiano dirigido al foco de infección. ${ }^{13,14}$

Por lo tanto, el tratamiento y manejo oportuno de la sepsis 
en el embarazo presente en la UCI, debe ser un manejo vital para reducir la morbimortalidad y los costos costos en salud para la atención de los pacientes gestantes, por esta razón hacemos énfasis en el manejo oportuno e ideal para este tipo de pacientes.

\section{MÉTODO}

La presente revisión de la literatura se desarrolló con el objetivo de describir por medio de la evidencia científica disponible, aspectos referentes al proceso fisiopatológico y el tratamiento de la sepsis en el embarazo. Para esto se elaboró un proceso con etapas desarrolladas en la siguiente forma: a) definición del tema; b) su exploración y delimitación; c) búsqueda minuciosa de la información basada en los niveles de evidencia y grados de recomendación de la Scottish Intercollegiate Guidelines Network (SIGN); d) sistematización, análisis y evaluación de los artículos, y e) redacción de la revisión literaria, el artículo final y control de calidad del mismo.

La selección se inició con la lectura de los títulos de todas las publicaciones, luego se eligieron aquellas en los que se enmarcó el tema de infección en gestantes centrándose en las causas, la mortalidad en UCI investigando las fallas terapéuticas que expliquen el aumento del porcentaje, el tipo de manejo en UCI y por último los efectos sobre el feto en casos de sepsis.

Dentro de la búsqueda se tuvieron en cuenta los títulos que trataron sepsis y manejo de las maternas en UCI, así como los artículos con énfasis en los factores de riesgo, comportamiento de la sepsis en el embrazo y la prioridad del manejo médico.

\section{RESULTADOS}

La búsqueda arrojó un resultado de 98 referencias. En este proceso se identificaron 60 estudios de los cuales 29 no tenían directa relación con el tema, en 18 no se logró obtener el texto completo, solo el resumen, incluso haciendo contacto con algunos autores originales por medio del envío de correos electrónicos, 7 publicaciones no tenían la estructura de la atención de la paciente en cuidado crítico y los 6 restantes eran anteriores a 2005. Finalmente se obtuvieron $38(100 \%)$ artículos que cumplían todos los criterios de inclusión para la revisión, durante el período 2005 a 2017.

Además de esto se tomaron los términos previamente nombrados y establecidos, donde se realizó una búsqueda literaria de artículos de revisión $(\mathrm{n}=20)$ y libros $(\mathrm{n}=2)$; desde 1998 hasta 2015, de los cuales se tomaron los aspectos más relevantes y los aportes más importantes sobre la sepsis en el embarazo y el manejo de la misma en UCI; por esto el rango de tiempo se amplió con la finalidad de sustentar con más claridad la perspectiva fisiopatológica. Al finalizar la búsqueda se inició una etapa de sistematización de la información, para lo cual se realizó un análisis de la información examinando todos los artículos científicos y las revisiones, resaltando los datos de intervención médica para lograr su posterior descripción.

\section{ASPECTOS FISIOLÓGICOS Y} FISIOPATOLÓG I COS

La sepsis se caracteriza por una inflamación sistémica exacerbada dando respuesta a un estímulo infeccioso, que varía de acuerdo con la función del huésped, el estado nutricional, la virulencia del agente infeccioso y en relevancia la fuente de infección. ${ }^{3}$ Es importante nombrar que no todas las bacterias conducen a la sepsis, pues todos tenemos bacteriemia a diario especialmente en la cavidad oral, pero es ocasional que se desarrolle. ${ }^{15,16}$

De acuerdo con la definición específica se ve la importancia de nombrar los criterios de disfunción orgánica que se evalúan de manera cuantitativa por la escala SOFA (Organ Failure Assessment Score), donde se evalúa. Cuando el paciente cumple con dos o más criterios se considera como disfunción orgánica, y si se comprueba una infección ya se diagnostica como sepsis. ${ }^{1}$

Gracias a este estimulo todas las toxinas del agente infeccioso estimulan la liberación de las células encargadas de la defensa, sobre todo las citoquinas proinflamatorias encargadas de activar el tejido endotelial para así producir los efectos de sepsis. Esta se desarrolla cuando la infección es resistente a toda medida de defensa antibacteriana del huésped. Por lo regular se identifican dos etapas: preséptica (local) y séptica (generalizada), esta última es cuando la infección entra al torrente sanguíneo directamente. ${ }^{15-17}$

Al iniciar el proceso séptico el organismo responde con ciertos cambios que se reflejan en el recuento de plaquetas, temperatura, frecuencia cardiaca y demás síntomas que reflejan la presencia de inflamación, o sea la respuesta ante la presencia de un huésped. ${ }^{18}$

En las embarazadas no se dispone de buenas bases científicas para establecer con precisión el pronóstico, ya que el sistema inmunitario fetal no se ha completado y hay variabilidad en la respuesta a los estímulos infecciosos. ${ }^{3} \mathrm{Se}$ debe tener en cuenta que el ácido láctico es un subproducto directo del metabolismo anaeróbico, presente no solo en la sepsis sino también en choque séptico, lo cual conduce a hipotensión arterial y disfunción en la microgranulación, disminuyendo la extracción de oxígeno en los tejidos periféricos con aumento en la producción y eliminación de ácido láctico, ocasionando el desarrollo de una acidosis láctica. ${ }^{19,20}$

Definida así la sepsis, existe una alta relación con la elevación del ácido láctico conduciendo a acidosis láctica 
que es una de las causas más comunes por la que las pacientes entran a la UCI obstétrica. Esta acidosis se puede presentar en las maternas con mayor facilidad a causa de que el bicarbonato sérico está en un nivel más bajo que en las no embarazadas, lo cual hace que la sean más susceptibles a una acidosis cuando se presenta una infección.

En cuanto a los cambios microbiológicos de las mujeres en estado de embarazo, algunos son anatómicos y otros hormonales, lo que genera una predisposición a la aparición de infecciones en el tracto genital inferior (TGI) o el urinario (ITU), siendo esta última una de las más comunes en el embarazo. Estas infecciones se asocian con las complicaciones del embarazo como el parto prematuro, rotura prematura de membranas, aborto espontáneo, corioamnionitis, endometritis posparto y enfermedad inflamatoria pélvica. ${ }^{4}$ Cuando se diagnostica una infección del TGI, es fundamental la identificación temprana de candidiasis, vaginosis bacteriana o tricomoniasis, con el fin de dar un tratamiento adecuado. ${ }^{4}$

\section{EPIDEMIOLOGÍA}

Siendo la sepsis una de las enfermedades más conocidas a nivel mundial Hipócrates la definió como el estado donde el huésped induce una respuesta inflamatoria como patógeno invasor con malos resultados. ${ }^{21}$ En cuanto a la definición laica, indica que la sepsis es un trastorno potencialmente mortal que aparece como respuesta del organismo ante un proceso de infección causando daños en sus propios tejidos y órganos. ${ }^{15,22}$

El ingreso más común a la UCI de ginecología y obstetricia son las infecciones del tracto urinario a menudo causadas por Escherichia coli y Staphylococcus aureus. Uno de los factores de riesgo más comunes por los cuales las madres gestantes contraen una infección es el parto prematuro, seguido de la rotura de membranas y fiebre intraparto. ${ }^{23}$

Si realizamos una asociación entre prematuridad y sepsis, la edad gestacional suele ser menor de 32 semanas lo cual genera una mayor probabilidad de contraer una infección por los cambios hormonales y físicos. ${ }^{23,24}$

Teniendo en cuenta que los recién nacidos deben desarrollarse en un medio intrauterino estéril y realizan su "colonización" cuando pasan por el canal del parto, en algunas ocasiones los microrganismos que se desarrollan en el tracto vaginal llegan a invadir la cavidad amniótica y el recién nacido puede tragar o aspirar líquido amniótico infectado. Se debe tener en cuenta que en los casos donde haya rotura prematura de membranas el riesgo es mayor para la infección ovular. Es por eso que se debe resaltar que si se disminuyera o eliminara la infección en embarazadas, se podría generar una reducción importante en la mortalidad neonatal, ya que en los neonatos pretérmino se genera una elevada mortalidad perinatal por sepsis. ${ }^{6}$

\section{I A G N ÓS T I CO}

Es fundamental el diagnóstico precoz al detectar algún síntoma en pacientes que posiblemente están infectadas, con el fin de disminuir la mortalidad a causa de la sepsis y la falla multiorgánica que se relaciona con ella. ${ }^{25}$ Es recomendable como método diagnóstico dos conjuntos de hemocultivos ${ }^{1}$ para obtener datos específicos. En las embarazadas se inicia con la toma sistemática del urocultivo al inicio, para evidenciar los agentes causales y la sensibilidad a los antibióticos. $^{4}$

\section{TRATA M IEN TO}

Existen dos paquetes de tratamiento para la reanimación según se proceda en las primeras 6 horas después de diagnosticada la sepsis o bien cuando el manejo se inicia entre 6 y 24 horas.

\section{REANIMACIÓN INICIAL}

Ante la sospecha de una sepsis severa se debe iniciar la terapia temprana en las primeras 6 horas. La meta es alcanzar una presión venosa de 8 a $12 \mathrm{~mm} \mathrm{Hg}$, presión arterial media mayor o igual a $65 \mathrm{~mm} \mathrm{Hg}$, eliminación de orina superior a $0.5 \mathrm{ml} / \mathrm{k} / \mathrm{h}$, asegurar un acceso venoso central y oxigeno mayor o igual a $70 \%$. Además se administran coloides y líquidos con el fin de evaluar la respuesta clínica del estado fetal. ${ }^{26-28}$

Cuando existen complicaciones y se hace necesaria la ventilación mecánica y el aumento de presión abdominal, se debe revisar la prescripción para las embarazadas donde la PVC y las presiones de la arteria pulmonar no son fiables al momento de relacionarlas. Los niveles suelen ser normales en pacientes con disfunción ventricular izquierda o edema pulmonar, pasa lo contrario en mujeres sin evidencia de edema pulmonar. ${ }^{26,27}$

El feto es susceptible a la hipotensión materna, por lo cual se deben administrar líquidos con rapidez, así mismo se recomienda la posición decúbito lateral izquierdo para poder revertir el síndrome de hipotensión supina que ocurre por la compresión de la vena cava inferior.

$\mathrm{Al}$ administrar cualquier medicamento es importante tener en cuenta la perfusión uterina y placentaria. Como la fiebre puede generar efectos adversos estructurales y funcionales sobre el feto, debe tratarse con rapidez con agentes antipiréticos y medios físicos. Además es importante la monitorización continua de la frecuencia cardiaca fetal. ${ }^{29,30}$ 


\section{USO DE ANTIBIÓTICOS}

De acuerdo con la evidencia se debe iniciar el antibiótico lo antes posible, basados en la historia para reconocer alergias, enfermedades subyacentes y condición clínica, recordando los patrones de resistencia a los antibióticos específicos de acuerdo con la prevalencia en la comunidad, para proceder con una terapia empírica. ${ }^{26}$ Cuando hay diagnóstico de sepsis grave o choque séptico, se debe garantizar una terapia de amplio espectro donde el organismo causante sea identificado al igual que su sensibilidad..$^{26,28}$

Con el resultado de los hemocultivos positivos en la paciente se debe tener precaución ya que la mayoría de las infecciones maternas son polimicrobianas. La elección del antibiótico para el manejo en embarazadas es complicado por varios factores ya que la dosificación debe ser limitada y se debe ver reflejado en todas las adaptaciones fisiológicas durante el embarazo. Pueden tener un impacto en la disponibilidad, concentración y su eficacia, sobre todo por el volumen de distribución, los cambios de distribución y de absorción afectando así los niveles del fármaco. ${ }^{13,26}$

Por lo general los antibióticos se eliminan por la orina. Los niveles séricos suelen ser más bajos por el embarazo, ya que la vida de algunos es más corta. En cuanto al estado del feto, el paso trasplacentario del antibiótico se produce, lo cual hace que lo principal es maximizar la efectividad y minimizar el daño del feto. ${ }^{31}$ La mayoría de las infecciones en las embarazadas son generadas por flora mixta, lo que incluye gram positivos, gram negativos y anaerobios. Por esto se hace énfasis en los antibióticos de amplio espectro utilizando dos o tres de ellos. La combinación más citada para muchos autores es ampicilina, gentamicina y clindamicina o metronidazol. ${ }^{20,26,32}$

En cuanto a la ventilación mecánica en la sepsis formulan tres recomendaciones: 1) emplear un volumen corriente de 4 a $6 \mathrm{~mL} / \mathrm{k}$ de peso; 2) que las presiones mesetas o llamadas plateau tengan un límite superior en un pulmón inflamado pasivamente; y 3) el PEEP se debe utilizar para evitar el colapso alveolar al finalizar la espiración. ${ }^{33,34}$

\section{FUENTE DE CONTROL}

Al iniciar las medidas de resucitación nombradas, la evaluación o seguimiento de la paciente debe centrarse en detectar el origen de la infección. La mayoría de las de naturaleza obstétrica son susceptibles a las medidas de control sobre el huésped. Cuando existe una corioamnionitis, es necesario realizar el parto lo antes posible sin importar la edad gestacional. ${ }^{35,36}$

\section{MEDIDAS COMPLEMENTARIAS}

Existe una campaña en diferentes hospitales de Estados Unidos en cuanto a la denominada sepsis sobreviniente, en la cual se aprueba el uso de corticoides como la hidrocortisona durante 7 días y en casos de choque séptico se adicionan vasopresores. Cabe resaltar que la hidrocortisona no está contraindicada en el embarazo cuando el parto prematuro sea probable y la vida del feto sea viable, recomendando como tratamiento prenatal la administración de la betametasona..$^{26,37}$

\section{EVALUACIÓN FETAL}

El monitoreo fetal se recomienda recordando que la frecuencia cardiaca permite valorar el episodio febril materno. Si se hace un tocodinámico se puede identificar si existe una taquistolia generada por la irritación del miometrio, ocasionada por la miometritis infecciosa..$^{21,35}$

La infección aguda puede generar contracciones uterinas ya sea con o sin modificaciones cervicales por la producción de endotoxinas. La mayoría de las veces hay respuesta positiva a la hidratación, pero en ocasiones puede iniciarse el trabajo de parto con dilatación cervical, a lo cual se responde con tratamiento tocolitico, sin olvidar que puede aumentar el riesgo de desarrollar edema pulmonar. Cuando el embarazo es menor de 34 semanas es recomendable realizar tratamiento tocolitico con sulfato de magnesio..$^{33,38}$

\section{CONCLUSIONES}

Las mujeres en estado de embarazo diagnosticadas con sepsis de cualquier causa, ya sea por infección urinaria o por mal manejo de una enfermedad pulmonar, deben atenderse de forma inmediata ya que en ocasiones suele ser asintomática y confundirse con manifestaciones propias del embarazo. Una vez diagnosticada es necesario iniciar el tratamiento que ataque al huésped aunque no se tenga claridad de cuál es, mientras los cultivos establecen qué microorganismos están proliferando. Cuando el diagnóstico es tardío, hay que trasladar a la paciente a UCI obstétrica para el seguimiento estricto de la madre y el feto. Si se considera necesario se realiza parto programado sin importar la edad gestacional. Se sugiere realizar más investigaciones en torno a la sepsis en el embarazo ya que el sustento científico es limitado.

\section{CONFLICTO DE INTERESES}

Los autores no declaran conflicto de intereses.

\section{DECLARACIÓN DE FINANCIACIÓN}

Nuestro proyecto no recibió financiación. 
1. Arsanios DM, Barragán AF, Garzón DA, Millán FC, Pinzón J, Isaza ER, et al. Actualización en sepsis y choque séptico: nuevas definiciones y evaluación clínica. Acta Colombiana de Cuidado Intensivo. 2017;17(3):158-183.

2. del Pilar Álvarez-Goris M, Zamora RS, Aguilar AAT, Calatayud ÁAP, Garduño JCB, Ángeles JS. Reconociendo la sepsis como causa directa de muerte materna en la Terapia Intensiva Obstétrica. Rev Asoc Mex Med Crit Ter Int 2016;30(3):178-182

3. Cordioli RL, Cordioli E, Negrini R, Silva E. Sepsis and pregnancy: do we know how to treat this situation? Rev Bras Ter Intensiva. 2013;25(4):334-44.

4. Briones Garduño JC, Viruez Soto JA, Vallejo Narváez CM, Vargas Arias RE, Ortiz Bolaños R, Díaz de León Ponce MA. Aislamientos microbiológicos: experiencia en obstetricia crítica. Rev Asoc Mex Med Crit y Ter Int. 2015;29(4):209-13.

5. Galvagno Jr SM, Camann W. Sepsis and acute renal failure in pregnancy. Anesth Analg. 2009;108(2):572-5.

6. Núñez JLE, Fernández MLSLP. Infección vaginal en gestantes y su influencia en la morbilidad y mortalidad perinatal. Multimed. 2017;21(2): 52-65

7. Albright CM, Ali TN, Lopes V, Rouse DJ, Anderson BL. The Sepsis in Obstetrics Score: a model to identify risk of morbidity from sepsis in pregnancy. Am J Obstet Gynecol. 2014;211(1):39 e1-. e8.

8. de León Ponce MAD, Garduño JCB, Santillán AAM, Vega CGB. La importancia de la medicina crítica en obstetricia. Rev Asoc Mex Med Crit y Ter Int. 2006;20(3):142-6.

9. Cohen J, Vincent J-L, Adhikari NK, Machado FR, Angus DC, Calandra T, et al. Sepsis: a roadmap for future research. Lancet infect Dis. 2015;15(5):581-614. doi: 10.1016/S1473-3099(15)70112-X.

10. Acosta $C D$, Knight $M$. Sepsis and maternal mortality. Curr Opin Obstet Gynecol. 2013;25(2):109-16. doi: 10.1097/ GCO.0b013e32835e0e82.

11. Surgers L, Valin N, Carbonne B, Bingen E, Lalande V, Pacanowski $\mathrm{J}$, et al. Evolving microbiological epidemiology and high fetal mortality in 135 cases of bacteremia during pregnancy and postpartum. Eur J Clin Microbiol Infect Dis. 2013;32(1):107-13. doi: 10.1007/s10096-012-1724-5.

12. Pineda C, Kaushik A, Kest H, Wickes B, Zauk A. Maternal sepsis, chorioamnionitis, and congenital Candida kefyr infection in premature twins. Pediatr Infect Dis J. 2012;31(3):320-2.

13. van Dillen J, Zwart J, Schutte J, van Roosmalen J. Maternal sepsis: epidemiology, etiology and outcome. Curr Opin Infect Dis. 2010;23(3):249-54. doi: 10.1097/QCO.0b013e328339257c.

14. Albright CM, Mehta ND, Rouse DJ, Hughes BL. Sepsis in Pregnancy: Identification and Management. J Perinat Neonatal Nurs. 2016;30(2):95-105. doi: 10.1097/JPN.0000000000000159.

15. Minasyan H. Sepsis and septic shock: Pathogenesis and treatment perspectives. J Crit Care. 2017;40:229-242. doi: 10.1016/j. jcrc.2017.04.015
16. Bonet M, Pileggi VN, Rijken MJ, Coomarasamy A, Lissauer D, Souza JP, et al. Towards a consensus definition of maternal sepsis: results of a systematic review and expert consultation. Reprod Health. 2017;14(1):67. doi: 10.1186/s12978-017-0321-6.

17. Kalin A, Acosta C, Kurinczuk JJ, Brocklehurst P, Knight M. Severe sepsis in women with group B Streptococcus in pregnancy: An exploratory UK national case-control study. BMJ open. 2015;5(10):e007976. doi: 10.1136/bmjopen-2015-007976

18. Singer M, Deutschman CS, Seymour CW, Shankar-Hari M, Annane $\mathrm{D}$, Bauer $\mathrm{M}$, et al. The third international consensus definitions for sepsis and septic shock (sepsis-3). JAMA. 2016;315(8):801-10.

19. Albright CM, Ali TN, Lopes V, Rouse DJ, Anderson BL. Lactic acid measurement to identify risk of morbidity from sepsis in pregnancy. American journal of perinatology. 2015;32(05):481-6. doi: 10.1001/jama.2016.0287.

20. Snyder CC, Barton JR, Habli M, Sibai BM. Severe sepsis and septic shock in pregnancy: indications for delivery and maternal and perinatal outcomes. J Matern Fetal Neonatal Med. 2013;26(5):5036. doi: 10.3109/14767058.2012.739221

21. Viteri González WR. Factores de riesgo de aborto séptico. Estudio a realizarse en el Hospital Martin Icaza de Babahoyo, peiodo 2015: Universidad de Guayaquil [Tesis]. Ecuador: Universidad de Guayaquil; 2016. 51 p.

22. Aarvold AB, Ryan HM, Magee LA, von Dadelszen P, Fjell C, Walley KR. Multiple organ dysfunction score is superior to the obstetricspecific sepsis in obstetrics score in predicting mortality in septic obstetric patients. Crit Care Med. 2017;45(1):e49-e57. doi: 0.1097/ CCM.0000000000002018

23. Andreu A, Ortega E, Planes AM, Salcedo S. Evolución de la sepsis perinatal por Escherichia coli en la era de la profilaxis del estreptococo del grupo B. Medicina Clínica. 2001;117(14):521-4.

24. Martin GS, Mannino DM, Eaton S, Moss M. The epidemiology of sepsis in the United States from 1979 through 2000. N Engl J Med. 2003;348(16):1546-54.

25. Molina RL, Easter SR, Venkatesh KK, Cantonwine DE, Kaimal AJ, Tuomala RE, et al. Defining Physiological Predictors of Peripartum Maternal Bacteremia. Am J Perinatol. 2015;32(14):1342-50. doi: 10.1055/s-0035-1565995

26. Guinn DA, Abel DE, Tomlinson MW. Early goal directed therapy for sepsis during pregnancy. Obstet Gynecol Clin North Am. 2007;34(3):459-79. doi: 10.1016/j.ogc.2007.06.009

27. Plante LA. Management of Sepsis and Septic Shock for the Obstetrician-Gynecologist. Obstet Gynecol Clin North Am. 2016;43(4):659-78. doi: 10.1016/j.ogc.2016.07.010

28. Brown KN, Arafeh JM. Obstetric sepsis: focus on the 3-hour bundle. J Perinat Neonatal Nurs. 2015;29(3):213-21. doi: 10.1097/ JPN.0000000000000115.

29. Lapinsky SE. Obstetric infections. Crit Care Clin. 2013;29(3):50920. doi: 10.1016/j.ccc.2013.03.006 
30. Labañino PP, Rodríguez RD, González MG. Listeriosis en una embarazada en Cuidados Intensivos Adulto. Presentación de caso. Rev Inf Cient. 2016;95(5):793-800.

31. Parfitt SE, Bogat ML, Roth C. Sepsis in Obstetrics: Treatment, Prognosis, and Prevention. MCN Am J Matern Child Nurs. 2017;42(4):206-9. doi: 10.1097/NMC.0000000000000341.

32. Eschenbach DA. Treating spontaneous and induced septic abortions. Obstet Gynecol. 2015;125(5):1042-8. doi: 10.1097/ AOG.0000000000000795.

33. Nares-Torices MA, Hernández-Pacheco JA, Estrada-Altamirano A, Lomelí-Terán JM, Mendoza-Calderón SA, Flores-Cortés MI, et al. Manejo de sepsis y choque séptico en el embarazo. Perinatol. Reprod. Hum. 2013;27(4):248-61.

34. Cuero-Vidal OL, Moreno-Sánchez DF, Torres-Bejarano MdM, MorenoDrada JA. Use of intravenous immunoglobulins in an obstetric patient with septic shock: Case report and review of the literature. Rev Colomb Obstet Ginecol. 2016;67(4):305-10. doi: 10.18597/rcog.1095

35. Galvão A, Braga AC, Gonçalves DR, Guimarães JM, Braga J. Sepsis during pregnancy or the postpartum period. J Obstet Gynaecol. 2016;36(6):735-43. doi: 10.3109/01443615.2016.1148679

36. Bowyer L, Robinson HL, Barrett H, Crozier TM, Giles M, Idel I, et al. SOMANZ guidelines for the investigation and management sepsis in pregnancy. Australian and New Zealand Journal of Obstetrics and Gynaecology. Aust N Z J Obstet Gynaecol. 2017;57(5):540-551. doi: 10.1111/ajo.12646

37. Ford JM, Scholefield H. Sepsis in obstetrics: cause, prevention, and treatment. Curr Opin Anaesthesiol. 2014;27(3):253-8. doi: 10.1097/ACO.0000000000000082.

38. World Health Organization. Statement on maternal sepsis. Geneva: World Health Organization; 2017. p. 4. 